\title{
Nanosecond infrared laser-induced precipitation of silver nanoparticles in glass
}

\author{
A. Wolak, ${ }^{1}$ M. Grabiec, ${ }^{1}$ O. Véron, ${ }^{2}$ J.-P. Blondeau, ${ }^{2}$ and K. Dzierżęga ${ }^{* 1}$ \\ ${ }^{1}$ Marian Smoluchowski Institute of Physics, Jagiellonian University, Reymonta 4, 30-059 Kraków, Poland \\ ${ }^{2}$ CEMHTI, CNRS/University of Orléans, Avenue de la Recherche Scientifique, 45071 Orléans cedex 2, France
}

Received May 20, 2013; accepted June 27, 2013; published June 30, 2013

\begin{abstract}
In this work, we report our results on the effect of Ag nanoparticles precipitation in soda-lime glass (doped with silver ions) during irradiation with nanosecond Nd:YAG laser pulses at $1064 \mathrm{~nm}$. Using on-line, optical extinction measurements and exploiting the localized surface plasmon resonance, we studied the evolution of nanoparticles' radius, their concentration and identified several characteristic phases of their growth. The studied nanoparticles differ in size and concentration depending on the local fluence within the laser beam. These processes are accompanied by a continuous decrease of the dielectric constant of the glass matrix which reflects the reduction of concentration of silver ions.
\end{abstract}

Silver nanoparticles (NPs) embedded in transparent dielectrics have been extensively studied due to their many potential applications in photonics. Glasses containing Ag NPs are good candidates for waveguides $[1,2]$, nanogratings [3], 3D data storage media [4] or optical limiters and switches [5]. These applications are based on unique linear and nonlinear optical properties of noble metal NPs originating from localized surface plasmon resonance (LSPR) [6]. The LSPR strongly depends on the size, shape and concentration of metallic NPs as well as on the properties of an embedding medium. Therefore, in-situ control of NPs synthesis in glass is of prime importance for the fabrication of materials with desired properties and their future applications in photonic devices.

One of typical syntheses of glass composite, containing metallic NPs, includes the introduction of appropriate metal ions into the matrix and subsequent thermal annealing $[7,8]$ or laser irradiation $[9,10]$ resulting in NPs precipitation via a chemical reduction process. It is commonly accepted that high-intensity laser pulses induce non-bridging oxygen (NBO) holes while the released electron is captured by the neighboring $\mathrm{Ag}$ ion. The local photo-reduction of $\mathrm{Ag}^{+}$and local laser heating induce the formation and diffusion of $\mathrm{Ag}$ atoms, their clustering and finally aggregation into nanoparticles [11]. Laser-driven reduction of metal ions and subsequent aggregation of NPs have been already achieved using $\mathrm{cw}$ laser sources [12, 13] or nano- [14, 15] and femtosecond laser pulses [16] of UV, visible and infrared wavelengths. The effect of irradiation strongly depends on pulse

\footnotetext{
*E-mail: krzysztof.dzierzega@uj.edu.pl
}

duration, laser fluence and radiation wavelength. For example, as a function of irradiation dose, the ns pulses at $532 \mathrm{~nm}$ initially promote the rise of the size and number of $\mathrm{Ag}$ NPs. A further increase of irradiation, however, results in their destruction due to photo-breakup and strong laser ablation. On the other hand, in the case of fs pulses, the heat transfer caused by conduction electrons to glass matrix is very limited, hence the diffusion and aggregation of $\mathrm{Ag}$ atoms need extra annealing or very high light fluence [16, 17].

In this paper we report on the space- and time-resolved measurements of laser-induced absorption in glass containing silver ions, with a transient pump-probe technique. The size and concentration of precipitating Ag NPs and their evolution is evaluated from the extinction spectra using the results of the Mie theory for spherical, non-interacting nanoparticles.

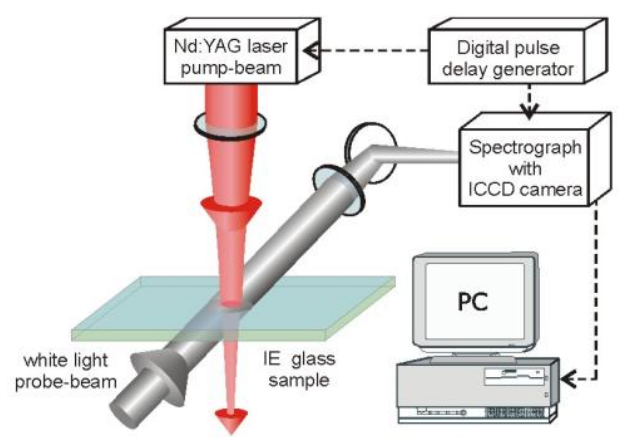

Fig. 1. Sketch of the experimental setup.

In our experiment we used soda-lime glass slides $(76 \mathrm{x}$ $25 \mathrm{~mm}^{2}$ x 1mm) with composition: $\mathrm{SiO}_{2}: \mathrm{Na}_{2} \mathrm{O}: \mathrm{K}_{2} \mathrm{O}$ : $\mathrm{CaO}: \mathrm{BaO}$ (71.67 : $14.93: 4.28: 5.4: 3.72 \mathrm{~mol} \%)$. The ion-exchanged (IE) samples were obtained by immersing the glass slides for 10 minutes in a molten salt bath of $10 \mathrm{~mol} \% \quad \mathrm{AgNO}_{3}: \mathrm{NaNO}_{3}$ at a temperature of $400^{\circ} \mathrm{C}$. During the immersion, some sodium ions, at the glass sub-surface layers, were replaced by silver ions from the solution. The thickness of these layers was investigated using scanning electron microscopy (SEM) in the back scattering mode [18] yielding an inhomogeneous profile with about $6 \mu \mathrm{m}$ penetration depth. Due to the high extinction of glass with NPs, in order to eliminate potential interference effects during optical 
measurements, one of these surfaces was polished to remove the IE layer.

The setup of our pump-probe experiment is sketched in Fig. 1. The IE samples were irradiated with an Nd:YAG laser beam (pump-beam) at a fundamental wavelength of $1064 \mathrm{~nm}(1.16 \mathrm{eV})$. The laser pulses of about $5 \mathrm{~ns}$ duration were delivered with a $10 \mathrm{~Hz}$ repetition rate and energy of $280 \mathrm{~mJ}$. The laser beam was weakly focused onto the sample surface to the spot of about $3 \mathrm{~mm}$ diameter (see Fig. 2d) yielding pulses of $3.1 \mathrm{~J} / \mathrm{cm}^{2}$ fluence. The irradiated area was simultaneously illuminated by a collimated white light beam (probe-beam) from a halogen lamp. The transmitted light was then imaged onto the slit of the spectrograph equipped with an image intensified charge-coupled (ICCD) camera. The ICCD and the optical collection system provided extinction measurements of the IE sample with a $60 \mu \mathrm{m}$ spatial resolution (across the pump laser beam). The measurements were performed with a spectral resolution of $1.5 \mathrm{~nm}$ in the range from 320 to $520 \mathrm{~nm}$ where LSPR in silver NPs is visible. The transmission spectra of the probe-beam were registered $100 \mu$ s after each laser pulse with $26 \mathrm{~ms}$ integration time. Such timing, controlled by a digital pulse generator, ensured that neither the pump laser light nor the potential luminescence from Ag NPs were collected.

Figure 2a shows the optical microscopic image of the IE sample as irradiated with 400 laser pulses. The colour of the whole irradiated area is dark yellow with a well defined brown rim appearing at the peripheries of the laser beam. Figures $2 \mathrm{~b}-\mathrm{c}$ present the extinction spectra, determined pulse by pulse, for the respective regions as they are marked in Fig. 2a. These spectra are already corrected for the extinction of the IE glass before irradiation, hence they correspond exclusively to the extinction of the IE layer.

As expected, no LSPR was observed before the irradiation of the IE samples. The LSPR appears above a specific number of deposited laser pulses, the earlier the higher the local fluence is. These LSPR spectra are characterized by different intensities, spectral positions and bandwidths. Nevertheless, after initial rapid variations, the LSPR becomes stable, although slight bleaching is observed for the central regions, i.e. with the highest laser fluence. Variations in the extinction can be explained by the precipitation of nanometer sized silver NPs, which was already confirmed by TEM measurements [15].

In the case of NPs much smaller than the optical wavelengths, the extinction is dominated by absorption and can be given by

$$
\operatorname{Abs}(\omega)=N \cdot \sigma_{a b s}\left(\omega, R, \varepsilon_{m}\right)
$$

where $N$ is the concentration of NPs and $\sigma_{a b s}$ is the absorption cross section for Ag NPs. According to the Mie theory, $\sigma_{a b s}$ for non-interacting spherical NPs is strongly dependent on the NP radius $R$ as well as on the dielectric constant $\varepsilon_{m}$ of the glass matrix [19].
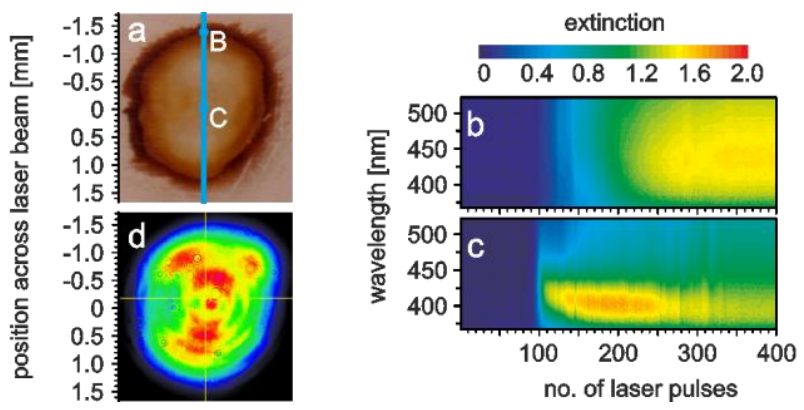

Fig. 2. Optical microscope image of the IE glass sample after irradiation by 400 laser pulses (a) and the profile of the laser beam (d). Pulse by pulse extinction spectra measured at the border (b) and the centre (c) of a laser beam at places marked as B and $\mathrm{C}$ in the optical image [panel (a)]. The blue line indicates a slit of the spectrograph.

We interpret our data using the model by Pinchuk et al. [20], which takes into account also the interband transitions in silver.

Figure 3 shows the absorbance spectra obtained for the central part (a) and the border (b) of the sample area irradiated with 170 and 106 pulses, respectively. The fitted function (red curves) yields the radius of NPs, their concentration and the dielectric constant of the IE glass matrix layer affected by silver atoms, ions and their clusters. The results of our measurements are consistent with TEM observations indicating NPs as small as 2-4nm in diameter. Moreover, the filling factor, based on NPs volume and concentration, is below 0.01 , which supports the assumption about non-interacting nanoparticles.
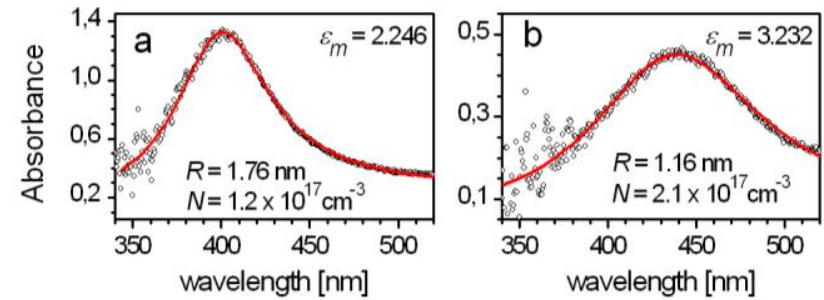

Fig. 3. Absorbance spectra as determined for the central part (a) and the border (b) of the IE sample irradiated by 170 and 106 laser pulses, respectively. Red curves represent the function fitted according to [19] with the parameter values presented in graphs.

The model function was fitted to the absorbance spectra obtained for individual areas of the irradiated IE sample after successive laser pulses. The fitted parameters are presented in Fig. 4 in the form of the spatio-temporal maps. The regions where either LSPR was not detectable or the signal to noise ratio was too low are marked in grey.

As seen in Fig. 4, NPs grow both in size and number beyond a specific threshold number of deposited laser pulses. Especially rapid growth is registered in the central 
parts of the sample, between about 100 and 120 pulses when NPs reach a radius of about $1.3 \mathrm{~nm}$. This growth can result from aggregation of atoms and clusters already embedded in the IE layer of glass. Further irradiation, up to about 250 pulse, makes these NPs still growing but at this time slowly, very likely at the expense of smaller particles since global concentration is decreasing. Such a process is commonly known as the Ostwald ripening [21]. After about 250 pulses the growth process is reversed and NPs are becoming smaller while their concentration is slightly increasing. This might be explained by laser ablation of large NPs due to their enhanced absorption. While in the centre of the laser beam NPs undergo many modifications, they seem to get stable after initial rapid growth at its border. In general, at the border of the laser beam they are about half the size of those in the central parts but at the end of irradiation their concentration is more than an order of magnitude higher. On the other hand, at the far borders (beyond $\pm 1.2 \mathrm{~mm}$ ) there is a lot of deposited NPs which have very complex extinction spectra. Their interpretation, however, is beyond our theoretical model.

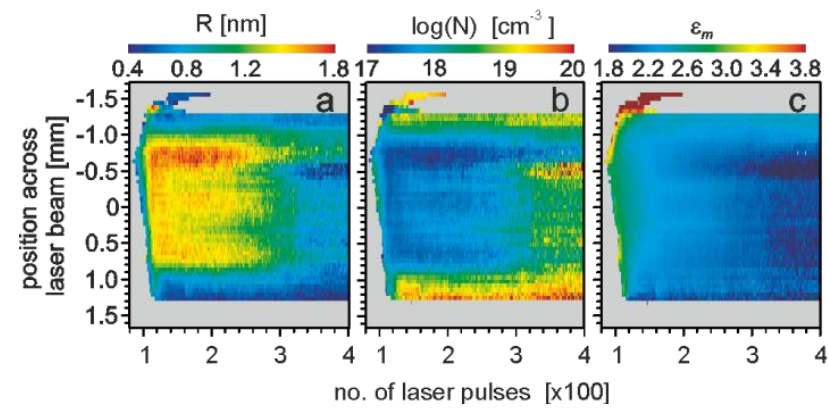

Fig. 4. NPs radius, concentration and the dielectric constant of the IE glass matrix determined by fitting the model function (according to [19]) to the absorbance spectra. In grey regions no function was fitted.

Along with NPs, the glass matrix, with the IE layer, also undergoes modification as demonstrated in Fig. 4. In particular, at the beginning of the precipitation process, the dielectric constant is significantly higher than that of the soda-lime glass (2.28-2.31 [22]) due to a high concentration of inclusions such as silver atoms, ions and their high polarisability. Then, with an increasing number of laser pulses, its value decreases monotonically in relation to a smaller and smaller number of silver atoms and clusters which are consumed by the growing NPs.

In summary, we have investigated the dynamics of formation of silver NPs in soda-lime glass doped with silver ions as a result of laser irradiation by nanosecond pulses of $1064 \mathrm{~nm}$. The precipitation of Ag NPs was studied on-line, pulse by pulse, with spatial resolution across the laser beam by optical extinction measurements. The results are interpreted in terms of localized surface plasmon resonance. It has been shown that NPs with a few nanometer radius and concentrations largely exceeding $10^{18} \mathrm{~cm}^{-3}$ are formed during irradiation in a way which strongly depends on local laser beam fluence. The NPs precipitation includes several stages, starting with the reduction of silver atoms and rapid aggregation of their clusters, to be followed by the Ostwald ripening and, finally, the destruction of the largest NPs by laser ablation. These processes are accompanied by modification of the glass matrix manifested in a continuous decrease of its dielectric constant reflecting the reduced content of silver.

We consider in-situ extinction measurements as a very simple and valuable method of controlling the synthesis of nanocomposites with metal NPs, supplementary to electron microscope imaging.

The research was carried out with the equipment purchased thanks to the financial support of the European Regional Development Fund in the framework of the Polish Innovation Economy Operational Program (contract no. POIG.02.01.00-12-023/08).

\section{References}

[1] G. De Marchi, F. Caccavale, F. Gonella, G. Mattei, P. Mazzoldi, G. Battaglin, A. Quaranta, Appl. Phys. A 63, 403 (1996).

[2] R.R. Gattas, E. Mazur, Nat. Photonics 2(4), 219 (2008).

[3] J. Gottmann, D. Wortmann, M. Hörstmann-Jungemann, Appl. Surf. Sci. 255, 5641 (2009).

[4] L. Canioni, M. Bellec, A. Royon, B. Bousquet, T. Cardinal, Opt. Lett. 33, 360 (2008).

[5] Y.P. Sun, J.E. Riggs, K.B. Henbest, R.B. Martin, J. Nonlinear Opt. Phys. Mater. 9, 481 (2000).

[6] D. Vollath, Nanomaterials: an introduction to synthesis, properties and applications (Weinham, Wiley-VCH 2008).

[7] X. Yang, W. Li, Y. Wei, W. Huang, Appl. Phys. A 90, 465 (2008).

[8] J.A. Jimenez, M. Sendova, T. Hartsfield, M. Sendova-Vassileva, Mat. Res. Bull. 46, 158 (2011).

[9] E. Trave, F. Gonella, P. Calvelli, E. Cattaruzza, P. Canton, D. Cristofori, A. Quaranta, G. Pellegrini, Nucl. Instrum. Methods in Phys. Res. B 268, 3177 (2010).

[10] A.L. Stepanov, V.F. Valeev, V.I. Nuzhidin, V.V. Bazarov, I.A. Faizrakhamov, Tech. Phys. 54, 1504 (2009).

[11] P.W. Wang, Appl. Surf. Sci. 120, 291 (1997).

[12] A.P. Nacharov, N.V. Nikonorov, X. Jiang, A.I. Sidorov, V.A. Tsekhomskii, Glass Phys. Chem. 34, 693 (2008).

[13] Y. Battie, N. Destouches, L. Bois, F. Chassagneux, A. Tishchenko, S. Parola, A. Boukenter, J. Phys. Chem. C 114, 8679 (2010).

[14] J. Zhang, W. Dong, J. Sheng, J. Zheng, J. Li, L. Qiao, L. Jiang, J. Cryst. Growth 310, 234 (2008).

[15] M. Grabiec, A. Wolak, O. Véron, J.-P. Blondeau, N. Pellerin, M. Allix, S. Pellerin, K. Dzierżęga, Plasmonics 7, 279 (2012).

[16] H. Zeng, C. Zhao, J. Qiu, Y. Yang, G. Chen, J. Cryst. Growth 300, 519 (2007).

[17] H. Zheng, J. Qiu, X. Jiang, C. Zhu, F. Gan, J. Phys. Condens. Matter 16, 2901 (2004).

[18] J.-P. Blondeau, S. Pellerin, V. Vial, K. Dzierżęga, N. Pellerin, C. Andreazza-Vignole, J. Cryst. Growth 311, 172 (2008).

[19] M. Quinten, Optical Properties of Nanoparticle Systems (Weinheim, Wiley-VCH 2011).

[20] A. Pinchuk, G. von Plessen, U. Kreibig, J. Phys. D: Appl. Phys. 37 3133 (2004).

[21] W. Ostwald, Z. Phys. Chem. 34, 495 (1900).

[22] R.A. Synowicki, B.D. Johs, A.C. Martin, Thin Sol. Films 519, 2907 (2011). 